Note

\title{
Good practice: Facilitator training for the Saxion Top Talent Innovation Days
}

\author{
Nitie Mardjan, Marike Lammers, and Guus Vrauwdeunt \\ Saxion University of Applied Sciences, The Netherlands; n.p.mardjan@saxion.nl, \\ m.t.lammers@saxion.nl,r.g.h.vrauwdeunt@saxion.nl
}

Correspondence: $\underline{\text { n.p.mardjan@saxion.nl }}$

Received: 28 September 2018; Accepted: 13 December 2018; Published: 7 January 2019

Keywords: facilitator, cooperation, interdisciplinary, critical reflection, experiential learning, flexible training, co-creation

Note to reader: honors keywords (see introductory article of this issue) in italics in the text

\section{Introduction}

In recent years, Saxion University of Applied Sciences (The Netherlands) has developed a Top Talent program, currently consisting of 10 different honors programs and five excellence tracks. Students and teaching faculty are offered an introduction to participation in a Top Talent program with the 'Top Talent Innovation Days' (see appendix A), a two-day event requiring students to work in interdisciplinary groups on real-world problem situations. In this way, they are introduced to the philosophy and practice of the Top Talent programs. Participants (students and faculty) of previous Top Talent Innovation Days are prepared for their role as facilitators in interdisciplinary groups during the next edition of these Innovation Days. This preparation for facilitators is done in a three-day training course by experienced coaches. Thus, Saxion talent development is multi-layered, including participants, facilitators, and coaches. Roles are assigned regardless of formal positions: students can be the facilitator or coach of faculty. The focus in the training course is learning how to facilitate the group process and reflection on individual and collective effectiveness in interdisciplinary cooperation. The objective of the Top Talent Innovation Days is twofold: learning how to approach real-world problems and learning how interdisciplinary cooperation can be effective. The learning from experiences is key to the success of the Top Talent Innovation Days. The program for this training and for the Top Talent Innovation Days was developed in cooperation by both students and faculty. 


\section{Why this good practice?}

The challenge is to provide all facilitators and, afterwards, the participants of the Top Talent Innovation Days (see appendix A) with insight in how to coach an interdisciplinary group in learning to work together more effectively.

This approach implies that it is possible, likely, and, to some extent, desirable that the process of cooperation derails. If cooperation is not smooth or far from smooth, there is room for improvement. And, that is where learning may take place, when obstructing factors can be addressed. The facilitator should intervene in the process and the role of the individual in the group; he or she should not intervene in the content or the quality of a new design as such that is not his or her responsibility. Facilitation is specifically aimed at the individual and collective effectiveness of cooperating in an interdisciplinary group with diverse backgrounds, i.e. a group of people who do not know each other at the start and have no affinity with the problem they are presented with (Davies \& Devlin, 2012).

The reflection in this two-day session is focused on the cooperation process and the role and contribution of the individual participant. It is important that participants learn to sense how a group is functioning and how each participant is involved in the process. Facilitators have experienced Top Talent Innovation Days in a previous year as participants and now act on a different level. As facilitators, they are part of the group as a functioning entity but with another responsibility, role, and focus. They are prepared to assume this role and responsibility through the so-called facilitator training. Coaches provide this training and support facilitators during the Innovation Days.

\section{Target group and target group size}

The Top Talent Innovation Days are meant for all students enrolled in Saxion Top Talent programs. Usually around 100 Top Talent students register as participants for the event. The facilitator training is aiming at Top Talent teaching faculty and students with very diverse backgrounds, that have the ambition to learn to effectively facilitate dynamic group processes (from here on indicated as 'trainees'). The size of the group in this facilitator training varies from 20 to 30 . The training is provided by five to six coaches. The formal training is offered through the Saxion Academy program and participants invest 60 hours.

\section{Educational design (structure)}

Here, the structure of the facilitator training is discussed. It strongly parallels the structure of the Top Talent Innovation Days but has a different purpose. During the Innovation Days, participants want to learn to effectively cooperate in an interdisciplinary setting, while, in the facilitator training, the participants want to learn how to effectively facilitate this cooperative process.

In preparation for the training, would-be facilitators get an assignment to prepare them for the activities: 'Make a note on what you want to learn and why you want to learn this, related to facilitating a group working on a wicked problem.'

The training consists of a 24-hour meeting including an overnight stay and is held outside the university. Shortly before and after this 24 -hour session, respectively, an introduction and a final prep meeting is scheduled. Trainees in the course have to cooperate in a new and 
unknown group, just as it is organized during the Top Talent Innovation Days. Three mixed groups of approximately 10 trainees are formed, varying in experience, background, and gender. The set-up of the Innovation Days is simulated, so that one can practice. Ample time is spent on interpretation and reflection. Formal functions are of no consequence: whether student, faculty, alumnus, or external person, you act as a facilitator-to-be. The training is organized and participants are coached by five to six coaches.

At the start, ample time is available for getting well acquainted. This part is important, since it provides the conditions for safe acting within a new group. It involves a set of activities that addresses backgrounds, motives, and current mood. The training is structured such that the trainee has options to choose from, depending on his or her assessment of the participants' needs. The program offers much diversity in activities (with energizers in between and drinks at the end of the days). Also, video-recording is used for feedback. The first day (and evening) involves a variation of activities with theory and practice of facilitating a team. The day is completed with an evaluation and an informal activity. During the second day, customized small group meetings are held for specific training of methods: techniques of coaching, reflection, role playing, and interventions in the group process. The second day is also completed with an evaluation. Facilitators constantly have to make decisions with regards to interventions and use the most effective method or tool to effectively facilitate or address the group process. There is no one recipe; it is a matter of grasping the essence of a group's (lack of) effectiveness and learning ability. This concept is thoroughly addressed in the training.

\section{Student assessment}

During the Innovation Days, the facilitators coach a fully new interdisciplinary group that works on a problem for a client. The coaching performed there is then also seen as proof of one's ability developed in the training.

\section{Experiences}

Some trainees say: 'The best course I ever did.' The course has provided them with real insight in the process of interdisciplinary cooperation. Everybody learns, regardless of formal function. Trainees recognize and appreciate the fact that their talents are used in a better way. Even if they, as facilitators, do not know anything about the content of a project on which the team is working, they can still help the group make progress. The trainees learn very much from reflection in-action and on-action directly during the activities of the group. The learning process of a trainee in the facilitator training resembles very much the learning process in the groups during the Innovation Days.

Teaching faculty who participated in this training understood that they can give students more freedom in a lesson or program while still achieving a learning result. Trainees appreciated the variation in content, the responsibility they were provided with, the equality within the group, and personal development.

\section{Time requirement for teachers}

Five coaches designed the training. For this designing, these coaches need meetings that take at least two days for each coach, spread over some months. For the initial edition, more hours are needed. The training takes three days plus a day for evaluation. Over the years, 
the set-up, the structuring, and the organization of the Top Talent Innovation Days have changed and so has the facilitator training. Key ingredients are still in place, but new insights and the involvement of fresh minds have helped to further develop both training and innovation days.

\section{Tips for teachers}

- Ask the trainees at the start of the training what they want to obtain from it and what they plan to bring.

- Allow ample time to get acquainted initially. This is key.

- Allow ample time for trainees to experience aligning with what is happening in the group they facilitate and allow them to develop their own style

- Don't tell the trainees what they have to do, but ask questions, so they get the idea themselves for the next step.

- Reflect on moments of 'eureka' for the trainees. Focus on these moments because these moments will be well-remembered.

- Require that trainees take some time to register what they learned, and which insights they obtained throughout the proceedings of the training.

- Don't worry; teaching faculty who are participating will also obtain new and valuable experiences.

\section{Tips for students}

By focusing on the process in the group and the cooperation instead of only on the method and the content, you can experience the learning environment much more intensively, and you may be surprised about the results.

\section{Transfer to other programs}

For this transfer, the following conditions are required:

-Participants come from different disciplines.

-Groups consist of a mix of teaching faculty and students.

-Have participants to work on real-world problems (where there is not one solution

available).

-Make sure to establish conditional safety within the group.

-Explicitly address the cooperation process.

Sometimes teachers of secondary education participate, and they are very positive about their experiences. This participation may be a first step to a transfer of this training to another educational program. The future will demonstrate.

\section{References, additional information and relation with Circle of Talent Development}

\section{$\underline{\text { References }}$}

Checkland, P. (1988). Systems thinking, systems practice. Oxford: Wiley.

Davies. M. \& Devlin, M. (2007). Interdisciplinary Higher Education: Implications for Teaching and Learning. Internet Archive: 
https://web.archive.org/web/20071202175644/http://www.cshe.unimelb.edu.au/pdfs/Inte rdisciplinaryHEd.pdf

Vermaak, H. (2007). Plezier beleven aan taaie vraagstukken. Werkingsmechanismen van vernieuwing en weerbarstigheid. Vakmedianet.

\section{Additional information}

A video on the Facilitator training in 2016:

https://www.youtube.com/watch?v=sTwTvbaAp68

Blog of a participant in 2014:

https://janinabanis.wordpress.com/2014/07/06/what-so-what-now-what/

Background information on the Innovation Days and the Facilitator training:

https://saxion.nl/wps/wcm/connect/8ce7e8ba-fa9d-4ac4-8ed7-

5b68198beca0/Voorbereiding+facilitators+ttid+2016.pdf?MOD=AJPERES

Saxion Academy:

https://saxionacademy.saxion.nl/Apps/SA/site.nsf

\section{$\underline{\text { Relation with Circle of Talent Development }}$}

In the introductory article of this issue of JEHC, the 'Circle of Talent Development' has been described. Based on interviews with forty honors students and literature about honors programs, it reflects relevant activities of students regarding the development of their talents in an honors program. The author of this note has been asked to score the good practice in relation to the activities in the Circle of Talent Development. The meaning of the scores is: + = somewhat important; ++ = moderately important; +++ = very important; - = not applicable or irrelevant. The scores are intended to illustrate the strengths of a good practice for the reader. 
Figure 1: Circle of Talent Development in relation with the good practice 'Facilitator training for the Saxion Top Talent Innovation Days' with the teacher's scores

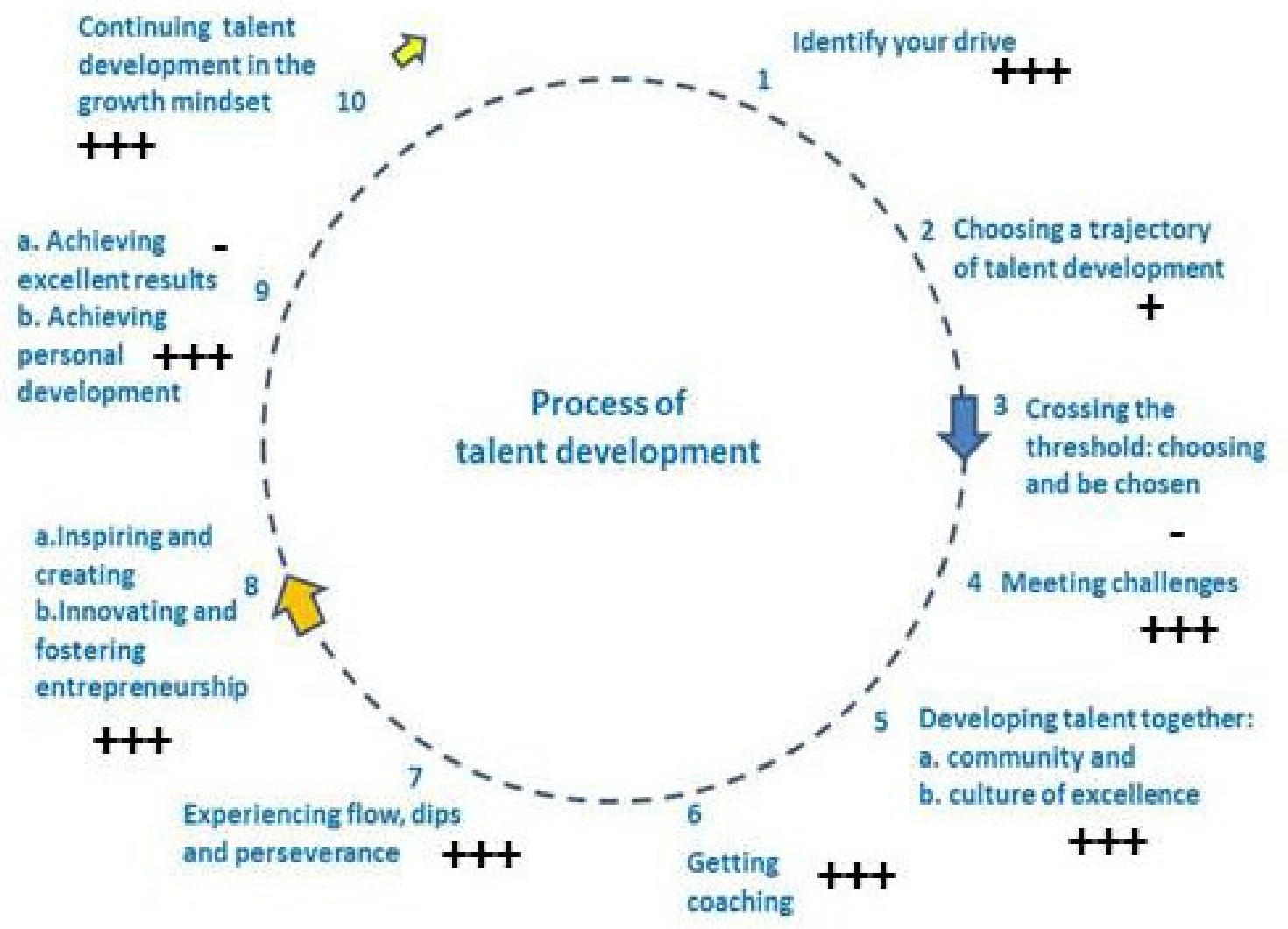

Additional information: step 6: Coaching also from peers! Mutuality in dialog (student vs. teacher); 9a: Results, learn a lot from failure!; 9b: Experience lead to insights in personal development.

Question to the teacher: What makes this good practice attractive for other teachers? The teacher of this practice argues: 'Teachers learn to become coaches and what different/new attitudes, skills, and behaviors are needed for coaches. Teachers experience it in this setting (experiential learning, learning community, learning by doing).' 


\section{Appendix A: Top Talent Innovation Days}

The Saxion Top Talent Innovation Days is a two-day event with about 150 persons participating. It involves two consecutive days, including an overnight stay. The goal is to work on real-world issues in an interdisciplinary group with the focus on the group process. These days are organized annually by the Saxion Top Talent team. The participants are arranged in groups of six to eight students and teaching faculty. About 20 facilitators are required for process facilitation. The groups are interdisciplinary and very diverse in composition, with students, teachers, and alumni. Groups are facilitated in the interdisciplinary cooperation process but not with regards to the approach of content, the problem at hand. An aim is that students and faculty learn to effectively cooperate with persons of different disciplines. Another aim is that the participants learn to formulate an assignment for themselves, get a view on the innovative aspects of it, and experience the learning environment of honors education.

An aim at the background is that the participants learn about the complexity of society. Socalled real-worldproblems, layered social problem situations for which not one solution exists, cannot be solved through a mono-disciplinary approach but need input from a diversity of disciplines (Vermaak, 2007). These problems require exploration from all perspectives, which allows for the development of appreciation for the perspectives of others (Checkland, 1988). That is not so easy because everybody has a filter. Facilitation stimulates different thinking and encourages participants to place themselves in the position of others and to understand the preferred approach of other disciplines. During this process, moments of reflection are programmed in different forms, and everybody gets feedback from everybody in the group.

Through cooperative learning from collective experiences with authentic themes and realworld assignments by actual clients, participants better understand the essence of honors education. Participating faculty learn what the power of honors education is.

There is no limit to the availability of wicked problems, contemporary real-world problem situations in society. The topics are not formulated as assignments but as themes by actual clients, who are present during the event. Every group starts by exploring the theme and then articulates the real-world problem to be approached. At least two groups are involved in each theme. The assignments originate in this way from authentic situations and involve an authentic client.

Sometimes, a new and unknown theme is surprising because the group cannot use traditional methods and has to find a new approach. Two examples of themes are 'The costs of healthcare are rising beyond what we can afford' or 'Traffic jams in The Netherlands are an economic headache'.

The results of the work in the groups is presented in pitches at the end of the two-day meeting. The clients of the assignments are also involved in these presentations. In these pitches, the final result, the approach, and the group's cooperation process are addressed. This allows for the explicit formulation of both content-oriented proceeds as well as the extent of perceived effectiveness of interdisciplinary cooperation. Within the groups, the method of 'hot \& cold shower' is performed: one participant sits in the middle of the circle of participants. This participant is congratulated with the positive aspects that were 
observed in his performance, e.g. contributions to the group (hot shower). This participant then receives suggestions for aspects that he or she might want to improve upon (cold shower). Then, the next participant comes to the middle of the circle. Every participant takes his or her turn. These proceedings take approximately 30 minutes. 